\title{
Electrical stimulation of the posterior tibial nerve reduces neuropathic pain in patients with polyneuropathy
}

\author{
This article was published in the following Dove Press journal: \\ Journal of Pain Research
}

\author{
Ron Dabby ${ }^{1,2}$ \\ Menachem Sadeh ${ }^{1,2}$ \\ Ilan Goldberg',2 \\ Vitaly Finkelshtein ${ }^{1,2}$ \\ 'Department of Neurology, Wolfson \\ Medical Center, Holon, ${ }^{2}$ Sackler \\ School of Medicine, Tel Aviv \\ University, Tel Aviv, Israel
}

\begin{abstract}
Peripheral neuropathic pain (PNP) is caused by neuronal damage to the peripheral nervous system and usually affects the distal extremities. This open-label study examined the effect of short-term peripheral nerve stimulation (PNS) on individuals with PNP due to polyneuropathy. A total of 12 patients (mean age, $63.0 \pm 10.0$ years, $41.7 \%$ male) with daily bilateral PNP for at least 6 months (mean duration, $7.4 \pm 7.8$ years) received a total of six direct electrical stimulation therapies to the posterior tibial nerve at 3-4-day intervals. Eight patients completed the study and were included in the efficacy analysis. The average pain at baseline was $36.6 \pm$ 3.80 estimated by the Short-Form McGill Pain Questionnaire. After the last stimulation, pain was significantly reduced by $85.5 \%$ to $4.88 \pm 3.1$ ( $p=0.008)$. Six patients $(75 \%)$ had over $50 \%$ decrease in pain after the first stimulation therapy and $99.2 \%$ after the final stimulation therapy. The patients also reported statistically significant decreases in pain level (measured by visual analog scale), ranging from $54.85 \%$ to $87.50 \%$ after each of the stimulations as compared to the pain experienced prior to the stimulations. The procedure was safe without any serious adverse events. PNS has demonstrated excellent efficacy and improvement of PNP symptoms. Further studies in larger patient populations are warranted.
\end{abstract}

Keywords: peripheral nerve stimulation, posterior tibial nerve stimulation, peripheral neuropathic pain, quality of life

\section{Introduction}

Peripheral neuropathy is a debilitating condition caused by damage to the peripheral nervous system as a result of several precipitating factors including diabetes mellitus, infection, nutritional deficiencies, alcohol abuse, exposure to toxins, and cancer chemotherapy. The prevalence of peripheral neuropathy was estimated at approximately $2.4 \%$, increasing with age to $8 \%$, and its most common etiology is diabetes mellitus. ${ }^{1,2}$ The condition affects both genders at all ages, but symptoms are unique to each individual in terms of frequency, quality, and severity of pain.

The pathophysiology underlying peripheral neuropathic pain (PNP) is damaged, dysfunctional, or injured nerve fibers that lead to pathological electrical activity, changing nerve function at the site of injury and in the areas around it and resulting in complex, chronic pain. PNP usually affects the extremities and is often accompanied with symptoms such as numbness, tingling, burning sensation, and weakness. ${ }^{1,3}$ It has been estimated that neuropathic pain is probably second only to musculoskeletal pain as the greatest cause of chronic pain.

Effective management of PNP is difficult. Treatments are usually individually tailored, based on their symptoms and treatment compatibility. ${ }^{4} \mathrm{PNP}$ is often opioid
Correspondence: Ron Dabby

Department of Neurology, Wolfson

Medical Center, 62 Halochamim Street,

Holon 58100, Israel

Tel +97235028513

Fax+97235028827

Email dabbyr@netvision.net.il
Journal of Pain Research 2017:10 27|7-2723

2717 
resistant or requires higher doses of analgesics to achieve relief; sometimes it responds to antiepileptic and antidepressant medications acting on shared mechanisms. ${ }^{5,6}$ Many, if not most, surgical procedures have yielded limited success in the treatment of the pain.

Peripheral nerve stimulation (PNS) is the direct electrical stimulation of involved nerves outside of the neuroaxis and was the first clinical application of the gate control theory that stimulation of large diameter afferent fibers can "gate" the transmission of nociceptive activity. ${ }^{7}$ Used for over 40 years, this method, through both percutaneous approach and fully implantable peripheral stimulation or spinal cord stimulation, has provided a good option for the control of extremity pain in instances where conventional methods have failed and surgical treatment was deemed inappropriate. PNS has shown that implanted electrical stimulation is effective in reducing peripheral nerve causes of pain at several anatomic locations; however, it has only begun gaining popularity in recent years. $^{8}$

In most patients with PNP, pain starts in the plantar aspect of the feet and remains maximal at these areas. This area is innervated by the posterior tibial nerve. Therefore, we evaluated the acute effect of electrical stimulation of this nerve in individuals with PNP in the lower extremities. Based on the gate theory and clinical observations that electrical stimulation may have a widespread nonlocalized effect, our working hypothesis was that tibial nerve stimulation would be effective in alleviating PNP in polyneuropathy.

\section{Patients and methods Study setting and patients}

This was an open-label study conducted in an outpatient setting at the Wolfson Medical Center (Holon, Israel). The study was approved by the Wolfson Medical Center's Independent Ethics Committee, and all participants provided informed consent. All procedures performed in studies involving human participants were according to the ethical standards of the institutional and/or national research committee and the 1964 Declaration of Helsinki, and its later amendments or comparable ethical standards. Patients 18-80 years of age with daily bilateral PNP that had begun in the feet and was present for at least 6 months prior to study entry and had failed conservative treatments were included in the study. Additional inclusion criteria were mean average pain intensity of at least 6 , but less than or equal to 10 , on an 11-point numeric rating scale (NRS) recorded twice daily during the 2-week screening period. Potential participants were excluded from the study if their mean pain score had decreased by more than $30 \%$ during the screening period (regardless of whether their final score was higher than 6 on the NRS) and if they had any active cardiac or other implant, clinically significant neurologic disorders (except PNP), clinically significant or unstable medical or psychiatric condition, severe or unstable cardiovascular, pulmonary, gastrointestinal, hematological, hepatic, renal, or endocrine diseases (other than diabetes), severe peripheral vascular disease that could cause intermittent claudication or ischemic ulcers or limb ischemia, amputations or persistent ulceration due to diabetes mellitus, uncontrolled hypertension, any anticipated need for surgery during the study, increased risk of seizures, any malignancy in the past 2 years, or pain that could not be clearly differentiated from, or conditions that could interfere with the assessment of neuropathic pain.

\section{Study procedures}

Patients were administered a total of six stimulation therapies at 3-4-day intervals using KeyPoint Medtronic electromyogram (EMG) machine which is routinely used for nerve conduction and EMG studies. This is an off-label use for this device. Stimulation therapy consisted of temporary insertion of the stimulation lead into the patient's lower leg in proximity to the tibial nerve. Electrical pulses were transmitted from the pulse generator to the tibial nerve via the stimulation lead. Individual parameter settings were modified according to each patient's sensations and pain severity. Stimulus output of biphasic pulses was adjusted to deliver intermittent stimulation of the following parameters: frequency of up to $100 \mathrm{~Hz}$, average current of up to $10 \mathrm{~mA}$, amplitude of up to $3 \mathrm{~V}$, pulse width of $0.5 \mu \mathrm{s}$ to $2 \mathrm{~ms}$. The stimulation parameters were derived from previous tibial nerve stimulation studies in humans and animals. ${ }^{9,10}$ We adjusted the stimulation parameters in each patient to a level of mild non-painful paresthesias. The parameters depended on the proximity of the electrodes to the nerve and the individual sensitivity of each patient.

Each stimulation therapy included 3 minutes of stimulation followed by a 2-minute pause lasting 30 minutes in total. Although short electrical stimulation is sufficient to suppress pain, as seen in stimulation tests prior to spinal cord or peripheral nerve permanent implantation, we arbitrarily chose six cycles to establish the reproducibility of the treatment.

\section{Outcome measures and end points}

At baseline and upon completion of each of the six stimulation therapies, patients were asked to grade their pain level using the Short-Form McGill Pain Questionnaire 
(SF-MPQ). ${ }^{11}$ Pre- and poststimulation pain levels were also assessed by the patients using a visual analog scale (VAS). After each stimulation, patients were also asked to rate their overall satisfaction with the procedure on a scale of $1-4$ (1, very satisfied; 2 , moderately satisfied; 3 , slightly satisfied; 4 , not satisfied) and to rate the improvement of symptoms on a scale of $1-4(1$, significantly improved; 2 , moderately improved; 3, slightly improved; 4 , symptoms worsened). The patients completed a short version of the McGill quality-oflife questionnaire at baseline, after the fourth stimulation, and at the final study visit. ${ }^{11}$ Patients were asked about their pain symptoms, discomfort that may have been felt during the procedure, or other physiological or technical problems that may have arisen. They were asked to complete a pain diary for 2 consecutive days after each treatment. At the end of the study, the investigator subjectively rated the patients' comfort and contentment during the treatment, and the efficacy of the treatment on a scale of $1-5$ (1, poor; 2 , mild; 3 , good; 4, very good; 5 , excellent). The scale used in our study is conceptually similar to the clinical global impression (CGI) scale used to assess the overall impression of clinical status. ${ }^{12,28}$ The primary end point of the study was the severe adverse event rate within the procedure. The secondary end point was clinical success defined as the effect of the stimulation therapy on pain relief and on quality of life.

\section{Statistical analysis}

Patient baseline demographics, pain levels before and after each of the stimulations, and quality of life were summarized by descriptive statistics: for categorical variables the number of patients and their percentage are presented and for continuous variables the mean and standard deviation are presented. The change in pain levels between baseline and each stimulation therapy, the change in pain levels before and after each of the stimulations, and the change in quality of life between baseline and the end of treatment were analyzed using the signed-rank test. A $p$-value of $<0.05$ was considered statistically significant.

\section{Results}

\section{Patient demographics}

The demographic characteristics of the study population are presented in Table 1. A total of 12 patients (five males, seven females) with a mean age of $63.0 \pm 10.0$ years (age range: $47-84$ years) and a mean duration of neuropathic pain of $7.4 \pm 7.8$ years (range: 5 months to 30 years) were enrolled. Seven patients $(58.3 \%)$ had a medical history of diabetes, and three $(25.0 \%)$ reported vitamin B12 deficiency. The other
Table I Patient baseline demographics and disease characteristics

\begin{tabular}{ll}
\hline Characteristics & Study patients $(\mathbf{N}=$ I2) \\
\hline Age, years, mean \pm SD (range) & $63.0 \pm 10.0(47-84)$ \\
Females, $n$ (\%) & $5(41.7)$ \\
Neuropathic pain duration, years, mean \pm & $7.4 \pm 7.8(0.4-30)$ \\
SD (range) & \\
Pain events/day, n (\%) & $\mathrm{II}(91.7)$ \\
$\quad$ Continuous & $\mathrm{I}(8.3)$ \\
$>5$ & $7(58.3)$ \\
Medical history, n (\%) & $3(25.0)$ \\
$\quad$ Diabetes &
\end{tabular}

Abbreviation: SD, standard deviation.

two patients had idiopathic sensory neuropathy. The majority of patients (11/12) reported daily continuous pain and one reported over five events of pain every day. All patients had sensory complaints, such as burning, tingling, and numbness in the distal lower extremities. Neurological examination revealed variable degree of reduced temperature, pinprick, and vibration sensation, with normal proprioception. Muscle strength was normal.

Eight patients completed the study and were analyzed for efficacy of PNS treatment. Two patients dropped out because we could not achieve an effective nerve stimulation associated with paresthesias, probably secondary to ankle edema. One patient dropped out because he could not adhere to the treatment schedule. One patient dropped out because of non-compliance in filling out the pain monitoring diary.

\section{Changes in pain intensity during the study} Figure 1 shows pain levels according to the SF-MPQ at baseline and after each stimulation therapy. Average pain

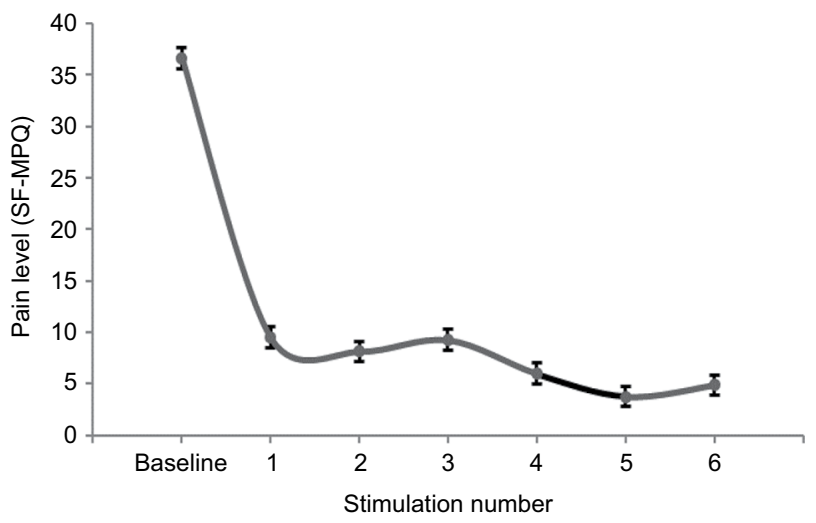

Figure I Pain level intensity during the study.

Notes: Pain level intensity was assessed using the SF-MPQ at baseline prior to the first study treatment and upon completion of each of the six stimulation therapies. Each point represents mean \pm standard error of the mean. The changes in pain levels between baseline and each of the stimulations were analyzed using the signed-rank test.

Abbreviation: SF-MPQ, Short-Form McGill Pain Questionnaire. 
at baseline was $36.6 \pm 3.80$. On average, after each of the stimulations, a statistically significant decrease of pain from baseline was observed. After the last stimulation, pain was significantly reduced by $85.5 \%$ to $4.88 \pm 3.1(p=0.008)$. In six of the patients ( $75 \%$ ), pain level decreased by more than $50 \%$ after the first stimulation therapy and by $99.2 \%$ after the final stimulation therapy. The patients also reported statistically significant reduced pain levels, measured by VAS, ranging from $54.85 \%$ to $87.50 \%$ after each of the stimulations as compared to the pain experienced prior to the stimulations (Figure 2).

\section{Overall efficacy of treatment}

At the end of the study, the investigator rated the efficacy of treatment and the change in symptoms (Figure 3). Efficacy of treatment was rated by the investigator as "excellent" in four (50\%) patients and "very good" in three (37.5\%) patients. Efficacy was rated as "poor" in one patient only.

Three patients (37.5\%) were rated by the investigator as having had excellent improvement in sensation during the study, two patients had very good improvement, and two patients had good improvement. One patient was rated as having had poor improvement in sensation.

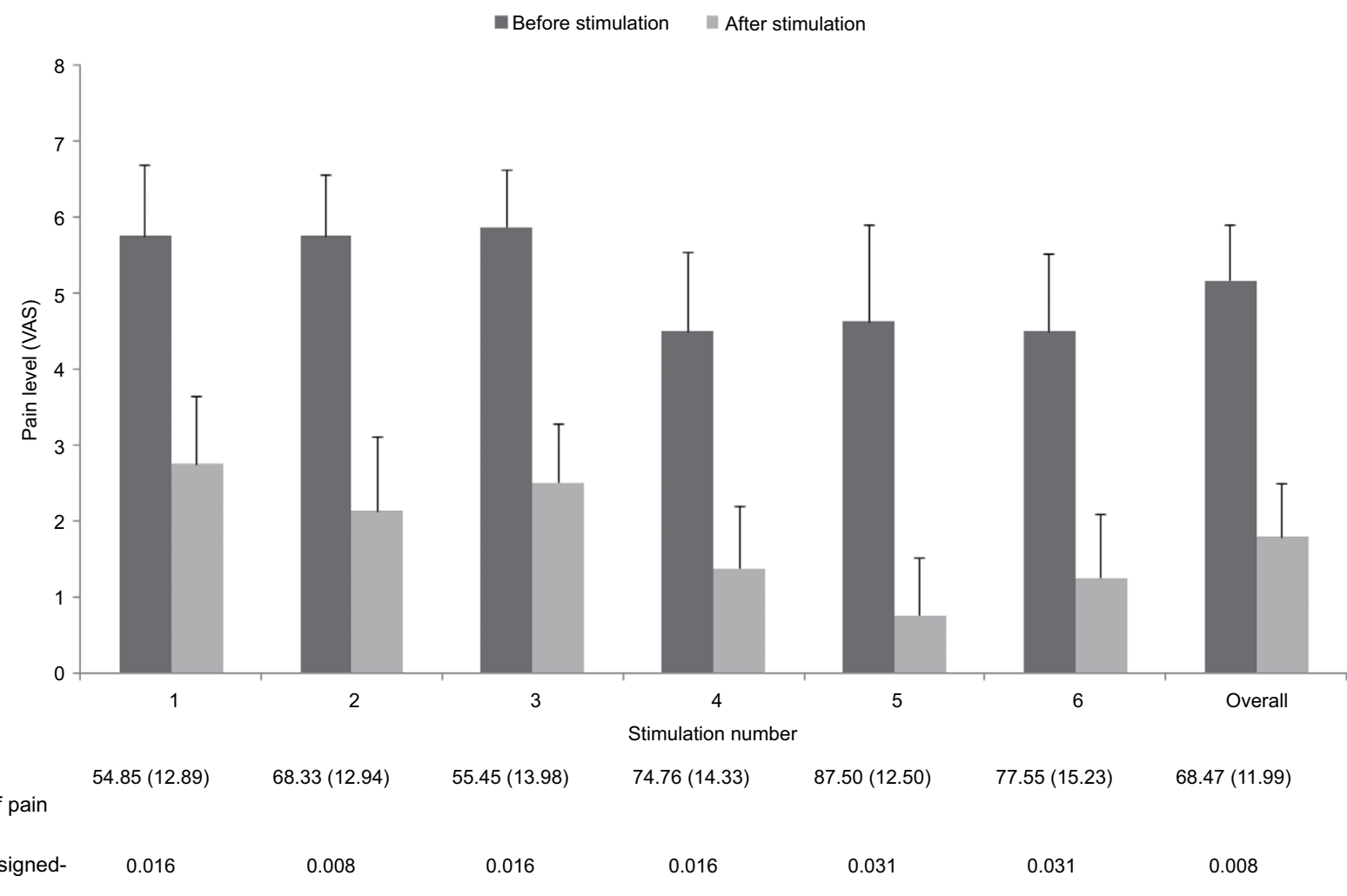

\begin{tabular}{|c|c|c|c|c|c|c|c|}
\hline $\begin{array}{l}\text { Percent } \\
\text { reduction of pain }\end{array}$ & $54.85(12.89)$ & $68.33(12.94)$ & $55.45(13.98)$ & 74.76 (14.33) & $87.50(12.50)$ & 77.55 (15.23) & $68.47(11.99)$ \\
\hline $\begin{array}{l}(\mathrm{SEM}) \\
P \text {-value by signed- } \\
\text { rank test }\end{array}$ & 0.016 & 0.008 & 0.016 & 0.016 & 0.031 & 0.031 & 0.008 \\
\hline
\end{tabular}

Figure 2 Pain level before and after each PNS treatment.

Notes: The patients reported their pain level using a VAS prior to and after each stimulation therapy. Each bar represents mean \pm SEM. The change in pain levels after each of the stimulations as compared to the pain before that stimulation was analyzed using the signed-rank test. The percentage of pain reduction and the $p$-value is shown below each of the stimulations.

Abbreviations: PNS, peripheral nerve stimulation; SEM, standard error of the mean; VAS, visual analog scale.

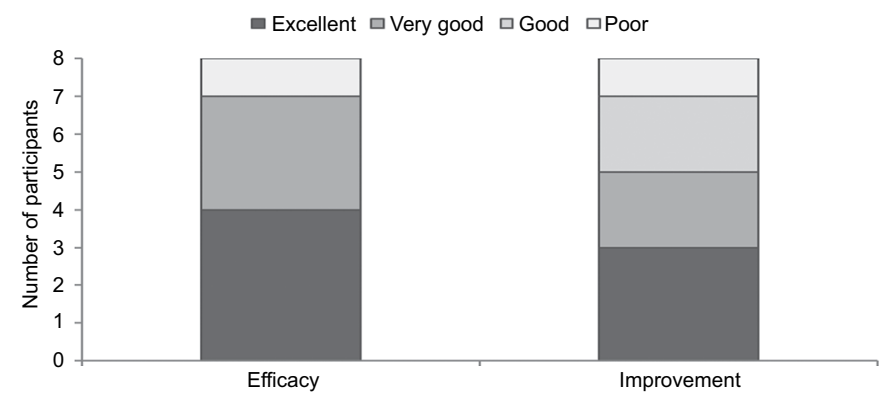

Figure 3 Investigator rating of efficacy and improvement of symptoms.

Notes: The changes in patient symptoms during the treatment and the efficacy of the treatment were rated by the investigator at the end of the study. The bars represent the number of patients in each category. 
Most patients also indicated that they were moderately satisfied or very satisfied with the treatment (Figure 4). Seven of eight patients (87.5\%) said they would use PNS treatment again and would recommend it to others.

On average, a nonstatistically significant increase of $31.8 \% \pm 18.4 \%$ in the patients' quality of life was demonstrated during the study.

All patients were followed after the end of the study. In all, the symptoms recurred within 2 weeks after the last neural stimulation.

\section{Safety}

Safety was assessed in all 12 patients who were enrolled in the study. Six patients (50\%) experienced seven adverse events (Table 2). None of the adverse events were serious, and all of the events resolved during the study period. Three of the adverse events were considered to be related to the device or to the procedure: one event of PNP exacerbation was considered related to the procedure. Heat sensation in the dorsal right leg and transient cold sensation were reported by the same patient and were considered to be related to the procedure. The event of heat sensation was also considered to be related to the device. At all consecutive treatments, all patients described their general health and neuropathic state as healthy. No postprocedure complications were reported.

\section{Discussion}

The current study examined the effect of a short-term PNS on individuals with PNP. Treatment resulted in significant alleviation of pain that was evident from the first stimulation therapy, reaching a reduction of $85.5 \%$ of the baseline pain by the sixth and final simulation therapy. Excellent efficacy and improvement of symptoms were demonstrated in most of the patients. The patients were generally satisfied with the treatment and reported that their symptoms had improved. Furthermore, the procedure was safe; no serious adverse events occurred during or after treatment and there were no infections or any other events that resulted in serious harm or disability.

PNS has undergone continuous development since the 1960s, when the first electrodes were implanted on the median and ulnar nerves. ${ }^{13}$ In the early 1970s, pain relief was demonstrated using percutaneous partially implanted PNS in individuals with posttraumatic neuralgias. ${ }^{14}$ Studies by Long ${ }^{15}$ revealed similar results with implanted PNS. Since then, PNS, through percutaneous electrical nerve stimulation and fully implantable PNS systems, has been used to treat a large variety of chronic pain conditions including postherpetic neuralgia and posttraumatic or postsurgical neuropathic pain that is related to underlying dysfunction of particular nerves, such as the infraorbital, supraorbital, or occipital nerves, classic migraine, transformed migraine presenting with occipital pain and discomfort, hemicrania continua, occipital neuralgia or cervicogenic occipital pain, complex regional pain syndrome, cluster headaches, chronic daily headaches, inguinal pain after herniorrhaphy, coccygodynia, and fibromyalgia. ${ }^{16}$ A 1998 meta-analysis demonstrated that electrical stimulation of peripheral nerves resulted in cumulative pain reduction in $82.5 \%$ of subjects, including those with posttraumatic neuropathy, neuroma pain, and diabetic neuropathy. ${ }^{17}$ Later studies likewise found a statistically significant reduction in pain after implantation and use of a peripheral nerve stimulator. ${ }^{18,19}$

Transcutaneous electrical stimulation of the tibial nerve in patients with incontinence was first described by McGuire et al. ${ }^{20}$ Later, Stoller ${ }^{21}$ adjusted this method by using percu-

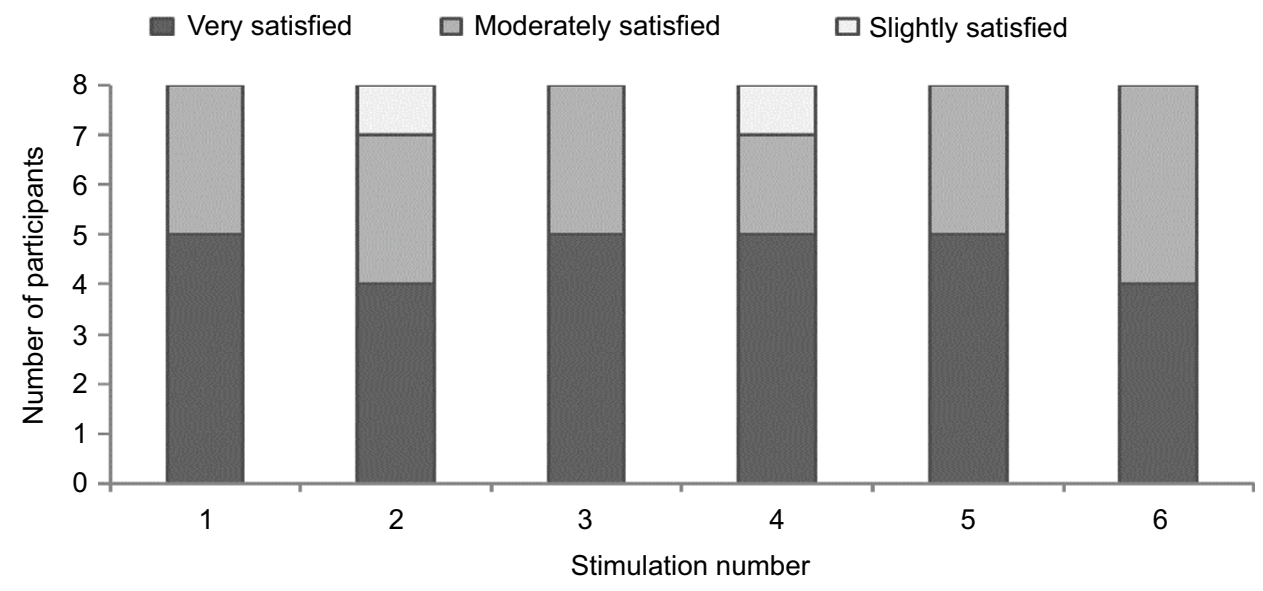

Figure 4 Patient satisfaction after each stimulation therapy.

Notes: The patients' overall satisfaction with the procedure was rated after each simulation therapy. The bars represent the number of patients in each category. 
Table 2 Adverse events

\begin{tabular}{ll}
\hline & Study patients $(\mathbf{N}=\mathbf{~ I 2 ) , \mathbf { n } ( \% )}$ \\
\hline $\begin{array}{l}\text { Number of patients with adverse } \\
\text { events }\end{array}$ & $6(50)$ \\
$\quad$ Headache & $2(16.7)$ \\
Exacerbated PNP & $2(16.7)$ \\
Nausea & I (8.3) \\
Heat in dorsal right leg & I (8.3) \\
Transient cold sensation & $\mathrm{I}(8.3)$ \\
\hline
\end{tabular}

Abbreviation: PNP, peripheral neuropathic pain.

taneous electrodes. Since then, several studies have shown encouraging results in patients with overactive bladder syndrome. The assumed mechanism is the modulation of the neuronal signals to the bladder, urinary sphincter, and the pelvic floor in an afferent mode..$^{22,27}$

Direct PNS in the lower extremities for the treatment of pain has been studied in several nerves, such as the saphenous, sciatic, and lateral femoral cutaneous nerve. ${ }^{23}$ Posterior tibial nerve stimulation in a relatively small number of patients with chronic pelvic pain showed promising results. ${ }^{24}$ The mechanism of action of neuromodulation remains rather unclear. The original explanation postulated that orthodromic stimulation of non-nociceptive large nerve fibers activates interneurons in the dorsal horn of the spinal cord which modulate the processing of nociceptive information. Another explanation is that electrical stimulation directly alters the excitability of peripheral nerve fibers, suppressing primary nociceptive afferents. It has also been suggested that PNS changes the concentration of local chemicals, such as endorphins, that are involved in pain generation. ${ }^{25,26}$ The advantages of PNS include its nondestructive nature and the fact that the procedure is reversible and can be turned off upon demand.

The field of PNS is now experiencing rapid growth in the number of patients undergoing such treatment, implanters, indications, and procedure types. Responding to a major need for safe and effective pain treatments and following a general trend toward less invasive interventions, PNS has the potential of becoming a premier pain-relieving modality that will be used instead of or in combination with existing more established approaches. ${ }^{22}$

The study's limitations include its small sample size (eight patients), short duration of treatment, and 33\% patient drop out.

\section{Conclusion}

The results of the current study have shown, within the limitations of the trial, that direct nerve stimulation is a safe and effective modality for alleviating neuropathic pain in patients with peripheral neuropathy. To the best of our knowledge, this is the first study that showed a beneficial effect of direct nerve stimulation in treating neuropathic pain in a generalized peripheral nerve disorder. Further investigations with larger numbers and longer duration may offer new perspectives for the treatment of PNP with neural stimulation.

\section{Acknowledgments}

The authors thank Dr. Sharon Furman-Assaf for assisting with the preparation of the manuscript. There was no funding for this study.

\section{Disclosure}

The authors report no conflicts of interest in this work.

\section{References}

1. Van Hecke O, Austin SK, Khan RA, Smith BH, Torrance N. Neuropathic pain in the general population: a systematic review of epidemiological studies. Pain. 2014;155(4):654-662.

2. Veves A, Backonja M, Malik RA. Painful diabetic neuropathy: epidemiology, natural history, early diagnosis, and treatment options. Pain Med. 2008;9(6):660-674.

3. Treede RD, Jensen TS, Campbell JN, et al. Neuropathic pain: redefinition and a grading system for clinical and research purposes. Neurology. 2008;70(18):1630-1635.

4. Zilliox LA. Neuropathic pain. Continuum (Minneap Minn). 2017;23(2): $512-532$.

5. Geber C, Baumgartner U, Schwab R, et al. Revised definition of neuropathic pain and its grading system: an open case series illustrating its use in clinical practice. Am J Med. 2009;122(10 suppl):S3-S12.

6. Dworkin RH, O'Connor AB, Audette J, et al. Recommendations for the pharmacological management of neuropathic pain: an overview and literature update. Mayo Clin Proc. 2010;85(3 suppl):S3-S14.

7. Melzack R, Wall PD. Pain mechanisms: a new theory. Science. 1965;150(3699):971-979.

8. Deer TR, Mekhail N, Provenzano D, et al; Neuromodulation Appropriateness Consensus Committee. The appropriate use of neurostimulation of the spinal cord and peripheral nervous system for the treatment of chronic pain and ischemic diseases: the Neuromodulation Appropriateness Consensus Committee. Neuromodulation. 2014;17(6):515-550. discussion 550.

9. Staskin DR, Peters KM, MacDiarmid S, Shore N, de Groat WC. Percutaneous tibial nerve stimulation: a clinically and cost effective addition to the overactive bladder algorithm of care. Curr Urol Rep. 2012;13:327-334.

10. Choudhary M, van Mastrigt R, van Asselt E. Inhibitory effect of tibial nerve stimulation on bladder neurophysiology in rats. Springerplus. 2016;5:35-43.

11. Melzack R. The short-form McGill Pain Questionnaire. Pain. 1987;30(2):191-197.

12. Demyttenaere K, Desaiah D, Petit C, Cornelian J, Brecht S. Patientassessed versus physician-assessed disease severity and outcome in patients with nonspecific pain associated with major depressive disorder. Prim Care Companion J Clin Psychiatry. 2009;11(1):8-15.

13. Wall PD, Sweet WH. Temporary abolition of pain in man. Science. 1967;155(3758):108-109.

14. Sweet WH. Control of pain by direct electrical stimulation of peripheral nerves. Clin Neurosurg. 1976;23:103-111.

15. Long DM. Electrical stimulation for the control of pain. Arch Surg. 1977;112(7):884-888. 
16. Slavin KV. Peripheral nerve stimulation for neuropathic pain. Neurotherapeutics. 2008;5(1):100-106.

17. Long DM. The current status of electrical stimulation of the nervous system for the relief of chronic pain. Surge Neurol. 1998;49(2): $142-144$.

18. Eisenberg E, Waisbrod H, Gerbershagen HU. Long-term peripheral nerve stimulation for painful nerve injuries. Clin J Pain. 2004;20(3): 143-146.

19. Novak CB, Mackinnon SE. Outcome following implantation of a peripheral nerve stimulator in patients with chronic nerve pain. Plast Reconstruct Surg. 2000;105(6):1967-1972.

20. McGuire EJ, Zhang SC, Horwinski ER, Lytton B. Treatment of motor and sensory detrusor instability by electrical stimulation. $J$ Urol. 1983;129:78-79.

21. Stoller ML. Afferent nerve stimulation for pelvic floor dysfunction. Eur Urol. 1999;35(suppl 2):16.

22. Stanton-Hicks M, Panourias IG, Sakas DE, Slavin KV. The future of peripheral nerve stimulation. Prog Neurol Surg. 2011;24:210-217.
23. Pope JE, Carlson JD, Rosenberg WS, Slavin KV, Deer TR. Peripheral nerve stimulation for pain in extremities: an update. Prog Neurol Surg. 2016;29:139-157.

24. Van Balken MR, Vandoninck V, Messelink BJ, et al. Percutaneous tibial nerve stimulation as neuromodulative treatment of chronic pelvic pain. Eur Urol. 2003;43(2):158-163.

25. Goadsby PJ, Bartsch T. Central mechanism of peripheral nerve stimulation in headache disorders. In: Slavin KV, editor. Peripheral Nerve Stimulation. Basel: Karger; 2011:16-26.

26. Dickenson AH. Gate control theory of pain stands the test of time. $\mathrm{Br}$ J Anaesth. 2002;88:755-757.

27. Govier FE, Litwiller S, Nitti V, Kreder KJ Jr, Rosenblatt P. Percutaneous afferent neuromodulation for the refractory overactive bladder: results of a multicenter study. J Urol. 2001;165(4):1193-1198.

28. Cohen SR, Mount BM, Strobel MG, Bui F. The McGill Quality of Life Questionnaire: a measure of quality of life appropriate for people with advanced disease. A preliminary study of validity and acceptability. Palliat Med. 1995;9(3):207-219.

\section{Journal of Pain Research}

\section{Publish your work in this journal}

The Journal of Pain Research is an international, peer reviewed, open access, online journal that welcomes laboratory and clinical findings in the fields of pain research and the prevention and management of pain. Original research, reviews, symposium reports, hypothesis formation and commentaries are all considered for publication.

\section{Dovepress}

The manuscript management system is completely online and includes a very quick and fair peer-review system, which is all easy to use. Visit http://www.dovepress.com/testimonials.php to read real quotes from published authors. 